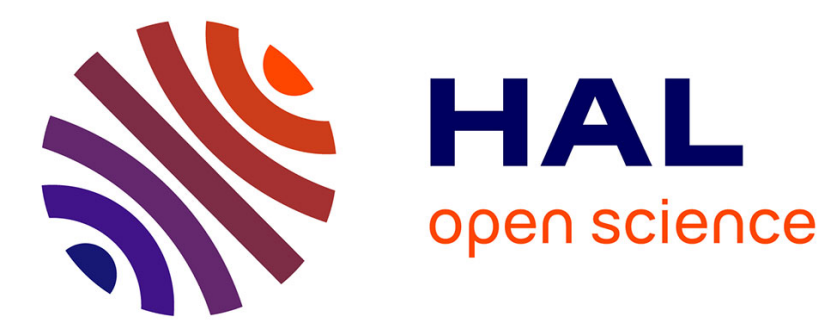

\title{
Fuzzy Logic Behavior of Quantum-Controlled Braitenberg Vehicle Agents
}

Rebeca Araripe Furtado Cunha, Naman Sharma, Zeno Toffano, François

Dubois

\section{- To cite this version:}

Rebeca Araripe Furtado Cunha, Naman Sharma, Zeno Toffano, François Dubois. Fuzzy Logic Behavior of Quantum-Controlled Braitenberg Vehicle Agents. 11th International Conference on Quantum Interaction (QI2018), Sep 2018, Nice, France. hal-01839282

\section{HAL Id: hal-01839282 \\ https://hal-centralesupelec.archives-ouvertes.fr/hal-01839282}

Submitted on 14 Jul 2018

HAL is a multi-disciplinary open access archive for the deposit and dissemination of scientific research documents, whether they are published or not. The documents may come from teaching and research institutions in France or abroad, or from public or private research centers.
L'archive ouverte pluridisciplinaire HAL, est destinée au dépôt et à la diffusion de documents scientifiques de niveau recherche, publiés ou non, émanant des établissements d'enseignement et de recherche français ou étrangers, des laboratoires publics ou privés. 


\title{
Fuzzy Logic Behavior of Quantum-Controlled Braitenberg Vehicle Agents
}

\author{
Rebeca Araripe Furtado Cunha ${ }^{1,2}$, Naman Sharma ${ }^{1,3}$, Zeno Toffano ${ }^{1,4}$, and \\ François Dubois ${ }^{5,6}$ \\ 1 CentraleSupélec, Université ParisSaclay, Gif-sur-Yvette 91190, France \\ 2 Federal University of Rio de Janeiro, Rio de Janeiro 21941-909, Brazil \\ ${ }^{3}$ National University of Singapore, 119077, Singapore \\ 4 Laboratoire des Signaux et Systèmes - CNRS (UMR8506), Gif-sur-Yvette, France \\ ${ }^{5}$ Conservatoire National des Arts et Métiers, Paris 75003, France \\ 6 Association Française de Science des Systèmes (AFSCET), Paris, France
}

\begin{abstract}
The behavior of agents represented by Braitenberg vehicles is investigated in the context of the quantum robot paradigm. The agents are processed through quantum circuits with fuzzy inputs, this permits to enlarge the behavioral possibilities and the associated decisions for these simple vehicles. The logical formulation Eigenlogic, using quantum logical observables as propositions and eigenvalues as truth values is applied in this investigation. Fuzzy logic arises naturally in this formulation when considering input states that are not eigenvectors of the logical observables, the fuzzy membership being the quantum mean value of the logical observable on the input state. Computer simulations permits visualization of complex behaviors resulting from the multiple combination of quantum control gates. This allows the detection of new Braitenberg vehicle behavior patterns related to identified emotions and linked to quantum-like effects.

[ver.3, June 11, 2018 - submitted to QI-2018]
\end{abstract}

Keywords: Quantum Robots · Fuzzy Logic · Quantum Gates · Braitenberg Vehicles . Emotion Analysis.

\section{Introduction}

With the recent improvements in quantum information, interest has been growing in the area of quantum robotics. This signifies using concepts from quantum computing to build and conceptualize systems capable of decision making. Simple robots capable of showing complex behavior have been introduced in Valentino Braitenberg's work on cybernetics proposing vehicle agents that show human-like emotions [1]. Here we aim to implement Braitenberg Vehicles (BV) using quantum-like circuits. Paul Benioff who was the first to propose the idea of a quantum Turing machine in 1980 was also the proponent of the theoretical principle of a quantum robot [2].

A quantum-like implementation of BVs has been undertaken in a previous research work [3], where Raghuvanshi et al. used the reversible quantum logical 
gate structure in building prototype BVs with Lego blocks. In the work presented here, in order to provide flexibility to the vehicle's behavior, we design fuzzy logic quantum-like circuits based on the quantum models proposed in [4].

Our goal here is to test the multiple combinations of quantum gates used in the control of BV by analyzing their complex behavior. For this purpose, we have developed a visual simulation tool illustrating BV's behaviors. This allows us to investigate new emotional behavior patterns not expected by a simple theoretical analysis used for for the description of BV in [1].

Mathematical models and simulations of individual and swarm automaton agents in response to environmental stimuli have attracted much interest for the understanding of complex behaviors of a group of animals. In [5], Kangan et al. developed a probabilistic control model (denominated ANIMAT) of mobile agents with biologically-inspired navigation, with the goal to mimic an ant colony. In this research it was made clear that the agent changes its state as a response to environmental stimuli and/or as a result of its own action on the environment, this observation can lead to path planning and intelligent-like emergent behavior of a group of agents. So agent behavioral models should take into account the uncertainty and the dynamics due to their environment.

A theoretical description of the model proposed here for the interaction of BV quantum robots with their environment is given in Section 2. This section details the mechanisms of transformation from the sensor input stimuli states to the control signals for the wheels of the BV (see Fig. Fig. 2).

In section 3 we provide a brief outline of the logical models used in the design of the BV quantum robots. These models are based on the Eigenlogic approach proposed in [4] and permit to characterize behaviors associated to fuzzy logic control.

We finish by illustrating the simulation results in Section 4 . In order to test the accuracy of our simulations, we first verify the behaviors predicted by the thought experiments proposed by Valentino Braitenberg [1]. This is done by observing how our simulator reacts to logical operators specifically designed for representing the vehicle's emotions of Fear, Aggression, Love and Exploration. After testing the BVs with these well established emotions, we test other Eigenlogic control operators such as i.e. the implication operator (see Dubois and Toffano in [4]). Finally using different operators in a variety of combinations gives us an interesting insight into the global emotional behavior of the vehicles.

\section{Modeling of the vehicle}

\subsection{Introduction to Braitenberg Vehicles}

In his book "Vehicles: Experiments in Synthetic Psychology" [1] Valentino Braitenberg describes various thought experiments using simple machines (Braitenberg Vehicles BV) that consist of sensors, motors and wheels. Similarly in our approach the quantum robot agents are represented by two controlled wheels at the rear of the $\mathrm{BV}$, with two sensors at the front. The sensors detect light 
produced by surrounding sources. The sensors can be be connected in different combinations to the wheels, and may have a positive or negative relation with the strength of the stimuli. These simple changes in configuration can lead to complex and surprising results in the agent behavior. Braitenberg terms this the "law of uphill analysis and downhill invention". According to this principle it is far easier to create machines that exhibit complex behavior based on simple connective structures than to try to derive their structures from behavioral observations and interpretations.

\subsection{Quantum approach for BV decision making}

To model the system we consider BVs with two sensors: SL in the upper left corner and SR in the upper right corner (see Fig. 2). The sensors detect light intensity and transform it into an input state vectors for the quantum circuit. The system then delivers as an output a fuzzy logical measure that is translated into wheel control by motors.

The computational block is composed of matrix operators designed using the quantum-like Eigenlogic method [4] discussed in section 3. We use two operators $\mathbf{F}_{L}$ and $\mathbf{F}_{R}$. Each one takes the inputs and delivers signals to the respective motors (left or right). This approach avoids to physically permute the wiring connecting sensors and motors in order to obtain different behaviors, it all can be handled by the computing device. The diagram in Fig. 1 resumes the inputoutput process.

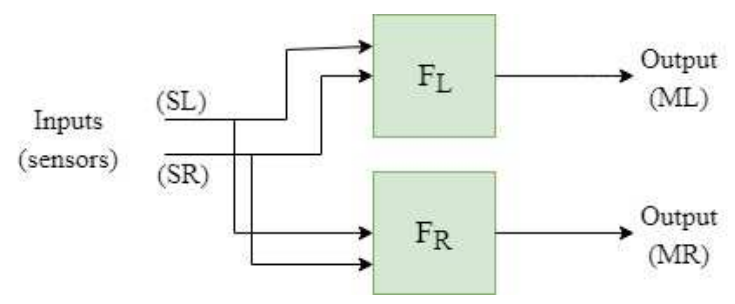

Fig. 1. Simplified diagram of the control circuit of a BV connected to the left and right input sensors SL, SR and to the output left and right wheel motors ML, MR.

The equivalent quantum formulation of the system consists in defining the input state vector presented in equation (1),

$$
|x\rangle=\left|x_{L}\right\rangle \otimes\left|x_{R}\right\rangle=\left|x_{L} x_{R}\right\rangle
$$

where the ket $|x\rangle$ corresponds to the combined input state of the bilinear system, formed by the Kronecker product of the individual input states $\left|x_{L}\right\rangle$ and $\left|x_{R}\right\rangle$ corresponding respectively to the left and right sensors. These vectors can be understood as qubits with a given orientation in Hilbert space. The output signal is obtained by the quantum mean value (Born rule) of the logical projection 
observable on the compound input state $|x\rangle$. It is the control function for the wheels. The quantities $\mu_{L}$ and $\mu_{R}$, for left and right wheel control, are:

$$
\begin{aligned}
& \mu_{L}=\left\langle x\left|\mathbf{F}_{L}\right| x\right\rangle \\
& \mu_{R}=\left\langle x\left|\mathbf{F}_{R}\right| x\right\rangle
\end{aligned}
$$

These quantities can also be interpreted as fuzzy membership functions [4]. In the following section we will show that these values are proportional to the angular speed of the wheels.

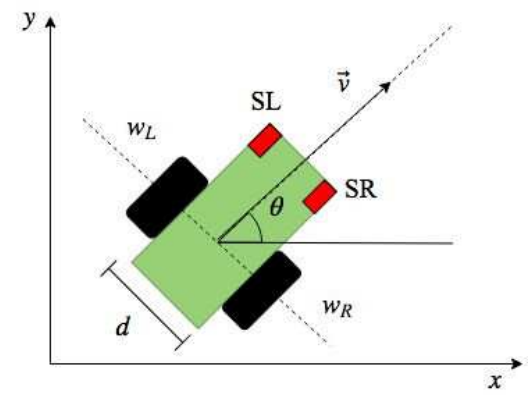

Fig. 2. Braitenebrg Vehicle orientation at speed $\boldsymbol{v}$ showing at the rear the left and right wheels WL, WR and at the front the light sensors SL, SR spaced at distance d.

\subsection{Output states and vehicle's motion}

Once the vehicle is submitted to the input state $\left|x_{f}\right\rangle$, it feeds it into the computation block as presented in the diagram of Fig. 1. The mean values for left and right are obtained as in equations (2) and (3), depending directly in the logical control operators $\mathbf{F}_{L}$ and $\mathbf{F}_{R}$. The wheel angular speeds $\omega_{L}$ and $\omega_{R}$ are calculated by applying the following relations:

$$
\begin{aligned}
\omega_{L} & =\mathrm{K} \cdot \mu_{L} \\
\omega_{R} & =\mathrm{K} \cdot \mu_{R}
\end{aligned}
$$

where $\mathrm{K}$ is a constant which allows us to tune the sensitivity of the vehicle to the stimuli. The $x$ and $y$ components of the vehicle speed vector (cf. Fig. 2) are determined by the following differential equations:

$$
\begin{aligned}
& \dot{x}=v_{x}=\frac{1}{2} R\left(\omega_{L}+\omega_{R}\right) \cos (\theta) \\
& \dot{y}=v_{y}=\frac{1}{2} R\left(\omega_{L}+\omega_{R}\right) \sin (\theta) \\
& \dot{\theta}=\frac{1}{d} R\left(\omega_{R}-\omega_{L}\right)
\end{aligned}
$$


where $R$ represents the $\mathrm{BV}$ wheel radius.

The period of time between every stimuli update is considered the unit of time of our system. Thus the vehicle position frame update equations are given by:

$$
\begin{aligned}
& x\left(t_{n+1}\right)=x\left(t_{n}\right)+v_{x}\left(t_{n}\right) \cdot\left(t_{n+1}-t n\right)=x\left(t_{n}\right)+v_{x}\left(t_{n}\right) \\
& y\left(t_{n+1}\right)=y\left(t_{n}\right)+v_{y}\left(t_{n}\right) \cdot\left(t_{n+1}-t n\right)=y\left(t_{n}\right)+v_{y}\left(t_{n}\right)
\end{aligned}
$$

\section{$3 \quad$ Topics in Eigenlogic}

Eigenlogic, [4] is a new quantum-like formulation in logic associating linear algebra and propositional logic. In this picture logical connectives are represented by observables and the truth values of the propositions are the eigenvalues of these observables. This permits to make a straightforward correspondence with quantum observables. As explained previously in Section 2, the computational block of our simulated vehicles is composed of two-argument logical observables.

\subsection{The Eigenlogic operators}

Because of logical completeness an $n$-argument (arity- $n$ ) binary logical system has a total of $2^{2^{n}}$ logical connectives. In Eigenlogic this is related to the number of possible compatible projection operators, which are commuting observables. For example, for a two qubit system defined in a 4-dimensional space, the number of possible logical connectives is $2^{2^{2}}=16$. In this case, we have to consider $2^{2}=4$ different distinct input cases corresponding to the 4 eigenstates of the logical system: $|00\rangle,|01\rangle,|10\rangle$ and $|11\rangle$. For each propositional case (eigenstate) we can assign a truth value which corresponds to one of the eigenvalues $\{0,1\}$.

For a given logical connective, we derive the corresponding logical observable by matrix interpolation methods using the eigenvalues and the associated projection operators as discussed in [6]. In the case of two logical input arguments $\mathrm{A}$ and $\mathrm{B}$, the basic logical projector operators and its negations are given in equation (11), where $\mathbb{I}$ is the identity operator.

$$
\begin{array}{ll}
\mathbf{F}_{A}=|1\rangle\langle 1| \otimes \mathbb{I} & \mathbf{F}_{\bar{A}}=(\mathbb{I}-|1\rangle\langle 1|) \otimes \mathbb{I}=|0\rangle\langle 0| \otimes \mathbb{I} \\
\mathbf{F}_{B}=\mathbb{I} \otimes|1\rangle\langle 1| & \mathbf{F}_{\bar{B}}=\mathbb{I} \otimes(\mathbb{I}-|1\rangle\langle 1|)=\mathbb{I} \otimes|0\rangle\langle 0|
\end{array}
$$

In general every logical connective can be expressed as a linear combination of the preceding observables using the conjunction connective. In logic this corresponds to the canonical form: disjunction of conjunctions SOP (Sum Of Products). In a 2-argument Eigenlogic system, one will consider the 4 conjunction observables corresponding to the rank-1 projection operators $\mathbf{F}_{A \cdot B}=\mathbf{F}_{A} \cdot \mathbf{F}_{B}$, $\mathbf{F}_{\bar{A} \cdot B}, \mathbf{F}_{A \cdot \bar{B}}$ and $\mathbf{F}_{\bar{A} \cdot \bar{B}}$. The scalar coefficients of the linear combination are the truth-values of the logical connective one wants to express. The linear interpolation used for the "implication" logical observable is given by: 


$$
\mathbf{F}_{A} \Longrightarrow B=1 \mathbf{F}_{A \cdot B}+1 \mathbf{F}_{\bar{A} \cdot B}+0 \mathbf{F}_{A \cdot \bar{B}}+1 \mathbf{F}_{\bar{A} \cdot \bar{B}}=\mathbb{I}-\mathbf{F}_{A}+\mathbf{F}_{A \cdot B}
$$

\subsection{Incorporation of Fuzzy Logic}

In order to better represent real situations, we consider that the stimuli of the BV's light sensors correspond to continuous variations of the light intensity. In our quantum formulation the vector eigenspace of a 2-argument Eigenlogic observables is the 2-qubit canonical basis $\{|00\rangle,|01\rangle,|10\rangle,|11\rangle\}$. State $|0\rangle$, corresponds to no light at all and state $|1\rangle$, corresponds to maximum luminosity.

Fuzzy logic deals with truth values that may be any number between 0 and 1 , where the truth of a proposition may range between completely false and completely true. In quantum mechanics one can always express a state-vector as a decomposition on an orthonormal basis. To make a correspondence with fuzzy characteristics we will consider not only eigenstates as input states but in general linear combinations of eigenstates, as shown in (13).

$$
\left|x_{f}\right\rangle=\alpha_{00}|00\rangle+\alpha_{01}|01\rangle+\alpha_{10}|10\rangle+\alpha_{11}|11\rangle
$$

The coefficients of the development can be considered as the weight of a particular logical state. The square module of each coefficient corresponds to the probability of being in that state and the sum of these probabilities will add up to one bacause of the othonormalization of the vector states. In our case, the compound fuzzy input state $\left|x_{f}\right\rangle$ is the Kronecker tensor product of the vectors $\left|x_{L}\right\rangle$ and $\left|x_{L}\right\rangle$ as defined in eq. (1). So we can write the state vectors:

$$
\begin{aligned}
& \left|x_{L}\right\rangle=\sqrt{1-p_{L}}|0\rangle+\sqrt{p_{L}}|1\rangle=\left[\begin{array}{c}
\sqrt{1-p_{L}} \\
\sqrt{p_{L}}
\end{array}\right] \\
& \left|x_{R}\right\rangle=\sqrt{1-p_{R}}|0\rangle+\sqrt{p_{R}}|1\rangle=\left[\begin{array}{c}
\sqrt{1-p_{R}} \\
\sqrt{p_{R}}
\end{array}\right]
\end{aligned}
$$

where the coefficients are function of the probabilities $p_{L}=\left|\left\langle x_{L} \mid 1\right\rangle\right|^{2}$ and $p_{R}=\left|\left\langle x_{R} \mid 1\right\rangle\right|^{2}$. The input state of the compound fuzzy system is then written as:

$$
\left|x_{f}\right\rangle=\left|x_{L} x_{R}\right\rangle=\left[\begin{array}{c}
\left(1-p_{L}\right)\left(1-p_{R}\right) \\
\left(1-p_{L}\right) p_{R} \\
p_{L}\left(1-p_{R}\right) \\
p_{L} p_{R}
\end{array}\right]^{\frac{1}{2}}
$$




\section{BV quantum robot simulation results}

We performed the simulation with different configurations of logical observables in the vehicle's computational unit. In this paper we illustrate only a small subset of the rich variety of possible combinations of observables. The graphical simulation interface allows the user to easily select the desired control operator corresponding to each wheel, as well as to add light sources and new vehicles in any time slot and location on the canvas ot the running simulation.

\subsection{Simulation environment}

As a result of the principle of the"Law of uphill analysis and downhill invention" [1], the behavior of these vehicles can become very complex. It is thus difficult to truly understand the response of these vehicles by the means of only thought experiments. Consequently, the natural method to analyze these vehicles is through computer simulations. The environment stimuli are light sources placed at different positions on the canvas. We opt for non punctual sources, where the intensity of each source is 1.0 inside the circle that delimits its border and decreases with the square of the distance to the border. The sources are considered as incoherent since they don't interact with each other, the total intensity at a given position is the sum of the intensities. Every vehicle has two sensors, one attached to its left and the other to its right side. The distance between both sensors is sufficient for the vehicle to distinguish the light intensities, in our model proportional to $p_{L}$ and $p_{R}$, in each of the corners. Different intensities will result in a change of the angle of the vehicle's speed vector. To each considered behavior corresponds a characteristic truth table given by the logical operator mean values on the eigenstates.

We will now describe the basic behaviors associated to BVs.

\subsection{Illustration of different emotional behaviors}

Fear: $\mathbf{F}_{\boldsymbol{L}}=\mathbf{F}_{\boldsymbol{A}} \quad \mathbf{F}_{\boldsymbol{R}}=\mathbf{F}_{\boldsymbol{B}}$ In this configuration, the quantum control gates simply connect the sensor readings to the wheel on the same side. Here, we see that the value of $\mu_{R}$ (resp. $\mu_{L}$ ) corresponds to $p_{R}$ (resp. $p_{L}$ ), because the logical control connective corresponds to the logical input. In Fig. 3, we clearly see the vehicle trying to avoid the stimulus in the case where the source is placed at a skew angle. In the case where the source is directly in front of the vehicle, the vehicle moves towards this source. The vehicle then comes to a rest in an area with almost no stimulus. This is the expected behavior for a BV possessing the emotion Fear.

Aggression: $\mathbf{F}_{\boldsymbol{L}}=\mathbf{F}_{\boldsymbol{B}} \quad \mathbf{F}_{\boldsymbol{R}}=\mathbf{F}_{\boldsymbol{A}}$ This configuration is similar to Fear with the difference that the quantum control gates connect the sensor output to the motor of the wheel on the opposite side. In Fig. 3, we observe a vehicle trying to collide with the source, regardless of the position of the source with 


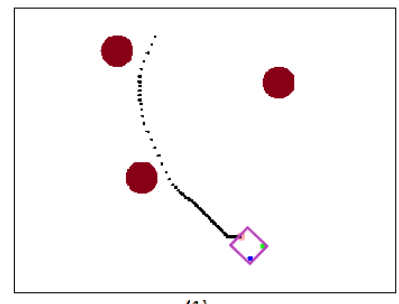

(1)
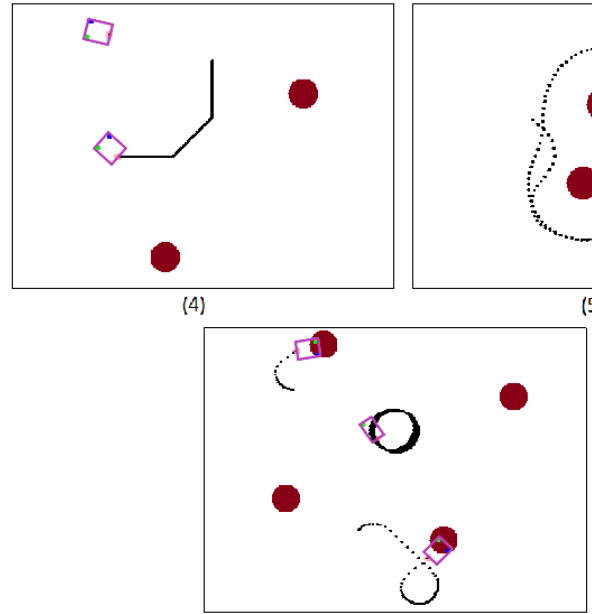

(6.a)

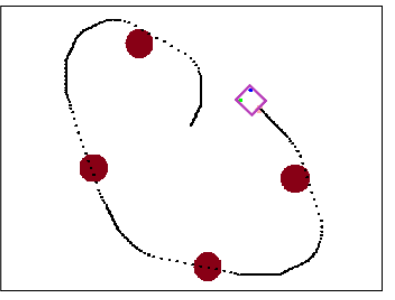

(2)
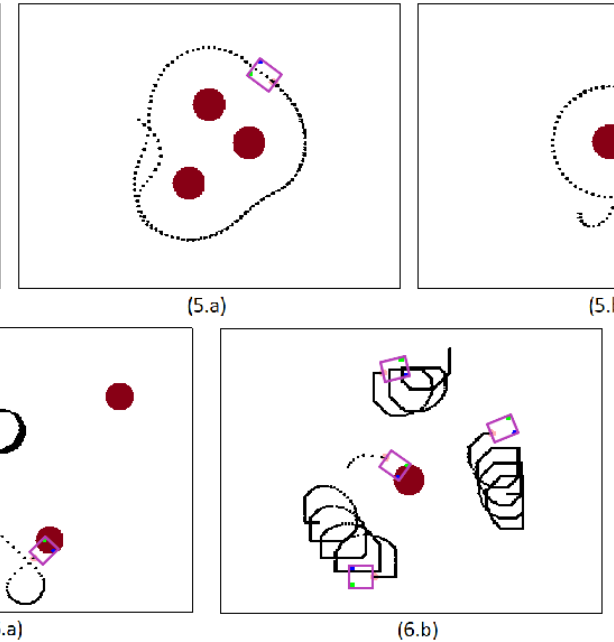

Fig. 3. Simulation screen-shots for (1)Fear, (2)Aggression, (3)Passion, (4)Boredom, (5)Worship, (6)Doubt

respect to the vehicle. Also in this case the vehicle comes to a rest in an area with almost no stimulus. This is the expected behavior for BV possessing the emotion Aggression.

Passion $\mathbf{F}_{\boldsymbol{L}}=\mathbf{F}_{\boldsymbol{B}} \not \boldsymbol{A} \quad \mathbf{F}_{\boldsymbol{R}}=\boldsymbol{F}_{\boldsymbol{A}} \not B$ According to simulations, as illustrated in Fig. 3, this vehicle remains at rest in the absence of stimulus. When it detects a light source, it moves towards it with increasing speed. The vehicle eventually hits the source and then comes to a rest. Observing the behavior of this vehicle, we compared this BV to the emotion Love [1]: the vehicle goes towards the light source and stops when it reaches the source. But in the present case it also shows aggressiveness because the speed increases as it gets closer to the source. Hence, we associate this BV emotion with Passion.

Boredom: $\mathbf{F}_{\boldsymbol{L}}=\mathbf{F}_{\boldsymbol{A}} \Longrightarrow \boldsymbol{B} \quad \mathbf{F}_{\boldsymbol{R}}=\mathbf{F}_{\boldsymbol{B}} \Longrightarrow \boldsymbol{A}$ In a thought experiment, one can expect that switching $(L \leftrightarrow R)$ the Eigenlogic operators of the Passion vehicle should lead to a result similar to that performed by the Explore vehicle 
described by Braitenberg [1], but with its own peculiarities. As one can see in Fig. 3, the vehicle remains at rest in the absence of light. In the presence of light it accelerates for a short period of time but then abruptly decelerates and comes to a stop before it actually hits the source. In the case another stronger source suddenly appears, the agent moves towards this source and then occupies a position at half-distance from both sources and facing away from the sources. Here, we interpret this BV emotion with Boredom.

Worship: $\mathbf{F}_{\boldsymbol{L}}=\mathbf{F}_{\boldsymbol{H} \boldsymbol{H}} \quad \mathbf{F}_{\boldsymbol{R}}=\mathbf{F}_{\boldsymbol{B}}$ Here, the control logical operator is the normalized projector version of the double-Hadamard quantum gate $\mathbf{H} \otimes \mathbf{H}$ :

$$
\mathbf{F}_{H H}=\frac{1}{2}(\mathbb{I}-\mathbf{H} \otimes \mathbf{H})
$$

The inputs of the vehicle can be represented by the truth-table given in Table 1 proportional to the respective fuzzy measures on the control logical operators $\mu_{L}=\left\langle x\left|\mathbf{F}_{H H}\right| x\right\rangle$ and $\mu_{R}=\left\langle x\left|\mathbf{F}_{B}\right| x\right\rangle$ :

Table 1. Worship emotion truth table

\begin{tabular}{cccc}
$\left|x_{f}\right\rangle$ & $\mu_{L}$ & $\mu_{R}$ & Behavior \\
\hline$|00\rangle$ & 0.25 & 0 & turns to the right slowly \\
$|01\rangle$ & 0.75 & 1 & turns to the left slowly \\
$|10\rangle$ & 0.75 & 0 & turns to the right \\
$|11\rangle$ & 0.25 & 1 & turns to the left
\end{tabular}

The vehicle keeps rotating around its own center in the absence of light. In the presence of light, it goes towards the source and starts to rotate around the source (or multiple sources when they are close together). This behavior can be seen in Fig. 3. We associate this vehicle emotion with Worship since the vehicles immediately start revolving around the light source once it senses it.

Doubt: $\mathbf{F}_{\boldsymbol{L}}=\mathbf{F}_{\boldsymbol{B}} \Rightarrow \boldsymbol{A} \quad \mathbf{F}_{\boldsymbol{R}}=\mathbf{F}_{\boldsymbol{X} \boldsymbol{O} \boldsymbol{R}}$ The projective version of the exclusivedisjunction (XOR) self-inverse Eigenlogic operator $\mathbf{Z} \otimes \mathbf{Z}[4]$ is used here:

$$
\mathbf{F}_{X O R}=\frac{1}{2}(\mathbb{I}-\mathbf{Z} \otimes \mathbf{Z})
$$

This operator provides a property that makes the vehicle turn around in circles, regardless of the presence or absence of stimuli. It is the other wheel control operator that completes the overall behavior of this vehicle. In the case considered here, we use the implication operator $\mathbf{F}_{B} \Rightarrow A$ as control operator for the left wheel. The truth table for this vehicle is given in Table 2. 
Table 2. Doubt emotion truth table

\begin{tabular}{cllc}
$\left|x_{f}\right\rangle$ & $\mu_{L}$ & $\mu_{R}$ & Behavior \\
\hline$|00\rangle$ & 0 & 0 & no movement \\
$|01\rangle$ & 1 & 1 & goes straight \\
$|10\rangle$ & 0 & 1 & turns to the left \\
$|11\rangle$ & 0 & 0 & no movement
\end{tabular}

This agent keeps rotating around itself in the absence of light. However, once it senses stimuli, it starts to roll towards it. Once it gets close to the source, it rotates outwards again and goes away from the source in a circular orbit. In some cases, the agent hits the source and comes to a stop (Fig. 3(6.a)). In particular cases, it starts rotating in a circle whose center itself is moving in a circle around the stimuli (Fig. 3(6.b)). We associate this vehicle with the emotional feeling of Doubt. The BV is unable to decide if it prefers light or not, and hence keeps coming to and going away from sources of light. In certain conditions, it decides to orbit around the source. In the case of sources on its both sides, it is unable to decide between the two and keeps switching from one to the other.

\section{Quantum Wheel of Emotions}

The concept of "wheel of emotions" introduced by Plutchik et al. [7] pictures the idea that a complex emotional state is the composition of elementary emotions. This picture can be interpreted in a quantum-like way using the quantum state vector $|\psi\rangle$. Each qubit (2-dimensional) quantum state can be mapped to a point on the surface of the Bloch unit sphere:

$$
|\psi\rangle=\cos \left(\frac{\theta}{2}\right)|0\rangle+e^{-i \frac{\phi}{2}} \sin \left(\frac{\theta}{2}\right)|1\rangle
$$

where $\phi$ and $\theta$ are the spherical angles. In order to simplify interpretation, the coefficients (associated with the degree of truth) multiplying the base states are taken as real numbers. The points of the vector are thus placed on a circle corresponding to a quantum wheel of emotions.

We summarized our simulation results by associating the different observed behaviors to a sector in the wheel as shown in Figure Fig. 4. Other emotions not presented in Fig. Fig. 3 have been simulated such as for example: Interest, Curiosity, Distraction, Fear, Worship and Sadness. The two latter ones have been obtained using a circuit that combines the standard quantum gates $\mathbf{H}$ and $\mathbf{C}_{\text {NOT. }}$.

The quantum wheel of emotions thus allows a continuous set of emotional states. A small perturbation in the angle of the input state $|\psi\rangle$ due to environmental factors, even if still inside the same emotional sector, will correspond to small changes in the vehicle's behavior. The measurement of the input state implies the collapse of $|\psi\rangle$ to a specific point of the wheel, and thus we can say 
that, in this aspect, the vehicle behaves as a quantum-like system. Furthermore, the fuzzy aspect of the system arouses naturally since the collapse can involve any state belonging to the continuous surface of the wheel. These observations can be compared to the observed similarities between neural network models and quantum systems. In particular, it has been suggested that it is possible to implement quantum learning algorithms dedicated to fuzzy qubits [8] where the weighted sums of inputs of a neuron correspond to the superposition of quantum states at the input of a quantum circuit and the quantum wave function collapse corresponds to the threshold activation of a neuron.

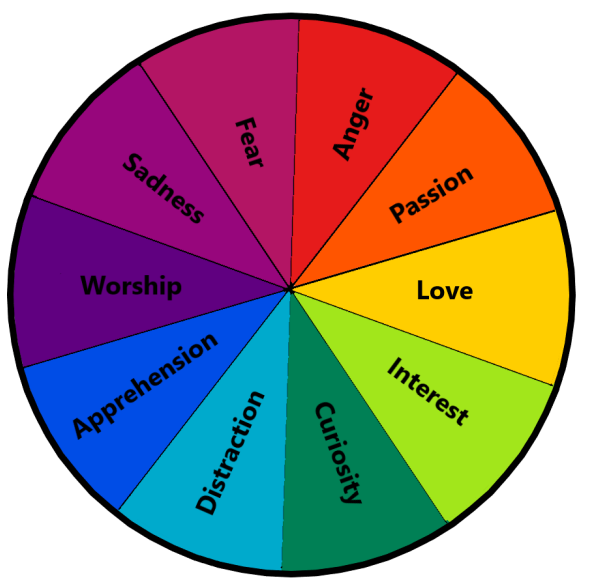

Fig. 4. Quantum Wheel of Emotions. For a given emotion, we associate quantum operators controlling the speed of the left and the right wheel respectively. (Anger) $F_{B}$, $F_{A}$; (Passion) $F_{B} \Longrightarrow A, F_{A} \Longrightarrow B$; (Love) $F_{\bar{A}}, F_{\bar{B}}$; (Interest) $F_{\bar{B}}, F_{\bar{A}} ;$ (Curiosity) $F_{A} \nRightarrow B, F_{B} \not A ;\left(\right.$ Distraction) $F_{A} \not B, F_{A}$ XOR $B ;\left(\right.$ Apprehension) $F_{B} \not A$ , $F_{A}$ XOR $B$; (Worship) $H \otimes H, F_{B}$; (Sadness) $C_{\mathrm{NOT}}, C_{\mathrm{NOT}}$; (Fear) $F_{A}, F_{B}$.

\section{Discussion and Conclusion}

The purpose of this research is to show the multiplicity of behaviors obtained by using fuzzy logic along with quantum logical gates in the control of simple Braitenberg Vehicle agents. The number of cases becomes intractable in simple theoretical approaches with increasing complexity. A computer simulation is mandatory and allows us to abstract the complexity by observing the motion of the vehicles and use it for illustrative purposes. At the same time, we see that by changing and combining different quantum control gates we can tune small changes in the vehicle's behavior, and hence get specific features around the main basic BV emotions of Fear, Aggression, Love and Explore. By tweaking these quantum gates, one can also obtain a vehicle that has a mixture of multiple emotions. 
Further extensions to this project can be imagined. Currently, when the vehicles collide, their respective control operators could change in order to reflect a quantum-like entanglement behavior due to interaction. It would be interesting to entangle the vehicles so that the behavior of one vehicle depends upon the current state of the environment from the perspective of other vehicles even after they separate after the collision. It could also be interesting to explore the Braitenberg vehicles using different types of stimuli (instead of only light) and sensors. Also a formalization of the quantum BV components as quantum neural networks could lead to new investigation strategies and could benefit researches in machine learning algorithms related to emotion analysis.

\section{Credits and Acknowledgements}

The first coauthor is under the Brafitec scholarship, CAPES Foundation, Ministry of Education of Brazil, Brasilia 70.040-020, Brazil.

We want to thank the AFSCET (Association Française de Science des Systèmes) for permitting us to present the idea of this work at the WOSC (World Organisation of Systems and Cybernetics) 2017 Congress in Rome Italy.

We are grateful to Francesco Galofaro of LUB for fruitful discussions on logic and semantics and their link to quantum theory and for pointing out that the late Professor Valentin von Braitenberg was one of the academic founding members of LUB (Libera Università di Bolzano/Freie Universität Bozen, Italy).

\section{References}

1. Braitenberg, V.: Vehicles - Experiments in Synthetic Psychology, MIT Press; Cambridge USA. (1986)

2. Benioff, P.: Quantum Robots and Environments as a first step towards a quantum mechanical description of systems that are aware of their environment and make decisions. Physical Review A, Vol.58, No.2, 893-904 (1998)

3. Raghuvanshi, A., Fan, Y., Woyke, M., Perkowski, M.: Quantum robots for teenagers. 37th International Symposium on Multiple-Valued Logic ISMVL (2007)

4. Dubois F., Toffano Z., Eigenlogic: A Quantum View for Multiple-Valued and Fuzzy Systems. In: de Barros J., Coecke B., Pothos E. (eds) Quantum Interaction Conference QI 2016. Lecture Notes in Computer Science, vol 10106. Springer (2017).

5. Kagan, E., Rybalov, A., Sela, A., Siegelmann, H.: Probabilistic Control and Swarm Dynamics in Mobile Robots and Ants. Biol. Tech. Knowl. Discov. Data Mining. January, 11-13 (2014)

6. Toffano, Z., Dubois, F.: Interpolating Binary and Multivalued Logical Quantum Gates. Proceedings of the 4th International Electronic Conference on Entropy and Its Applications. MDPI Proceedings, 2, 4, 152 (2018)

7. Plutchik, R.: The Nature of Emotions. Amercian Scientist, Vol. 89, n.4, 334-350 (2001)

8. Hannachi, M. S., Dong, F., Hirota, K.: Emulating Quantum Interference and Quantum Associative Memory Using Fuzzy Qubits. Proc. IEEE Int. Conf. Computational Cybernetics ICCC 2007, 39-45 (2007) 